\title{
Incontriamo Gesù. Studio sul documento della Commissione Episcopale Italiana sugli Orientamenti per l'annuncio e la catechesi $^{1}$
}

\author{
Incontriamo Gesù. A study of the document of the Italian Episcopal \\ Commission on the Guidelines for proclamation and catechesis
}

\begin{abstract}
This article discusses the document Meeting Jesus, presented by the Italian Episcopal Commission. In seeking to give guidelines for the proclamation and catechesis of the faith in Italy it becomes one of the most important documents in recent years that deal with the new evangelization that assists the whole Church in the announcing of the Gospel and the journey of faith.

The document highlights certain homogeneity in reading the cultural, social, ecclesial and pastoral situation in which the processes of formation and education are realized. This helps the development of the "catechetical needs" and the specific paths of diocesan and national guidelines. It also reveals the possibility of a comparison at the inter-diocesan level between formators and a mutual support in the evaluation of projects and activity. To realize the training of formators and of members of the diocesan teams "in the wise work of coordinating and leading" it is important to work on the same lines proposed by the Guidelines.
\end{abstract}

\section{Keywords}

Catechesis, catechist, proclamation, pastoral, formation of catechists, evangelization, education.

1 Conferenza Episcopale Italiana, Incontriamo Gesù. Orientamenti per l'annuncio e la catechesi in Italia, (d'ora in avanti $I G$ ), Edizioni Dehoniane Bologna, Bologna 2014. 


\section{Introduzione}

Il documento Incontrare Gesù presentato nel 2014 dalla Commissione Episcopale per la fede l'annuncio e la catechesi in Italia ${ }^{2}$ indica gli orientamenti per l'annuncio e la catechesi. Il testo è uno dei più significativi degli ultimi anni sulla nuova evangelizzazione, e presenta alla Chiesa i criteri per annunciare il Vangelo e il cammino di fede.

Papa Francesco rivolgendosi agli operatori pastorali e ai vescovi italiani, così si è espresso sugli Orientamenti per l'annuncio e la catechesi: «Abbiate fiducia che il popolo santo di Dio ha il polso per individuare le strade giuste. Accompagnate con larghezza la crescita di una corresponsabilità laicale; riconoscete spazi di pensiero, di progettazione e di azione alle donne e ai giovani: con le loro intuizioni e il loro aiuto riuscirete a non attardarvi ancora su una pastorale di conservazione - di fatto generica, dispersiva, frammentata e poco influente - per assumere, invece, una pastorale che faccia perno sull'essenziale. Teresa di Gesù Bambino, con un'espressione breve e la profondità dei piccoli "Amarlo e farlo amare", vede in Gesù il nocciolo dell'annuncio, lo stesso che la riflessione sugli Orientamenti per l'annuncio e la catechesi affronterà in queste giornate» ${ }^{3}$. Dalle parole di Francesco vengono indicate quattro dimensioni per rinnovare la missione evangelizzatrice: una corresponsabilità effettiva tra laici e pastori; il superamento di una pastorale tradizionalista non incentrata sull'«essenziale»; la testimonianza di amore riassunta nella citazione di santa Teresa di Gesù Bambino; l'annuncio e la catechesi come «nocciolo» dell'azione pastorale.

Il documento italiano intende ricordare alla Chiesa il suo compito missionario in linea con le tre note sull'iniziazione cristiana ${ }^{4}$, e con le tematiche relative all'annuncio e alla catechesi presenti negli orientamenti pastorali decennali della Commissione Episcopale Italiana. Il testo prende in esame integrandole

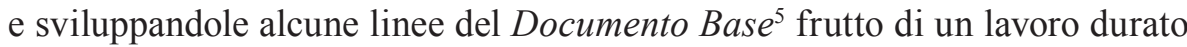
CEDAC).

2 Commissione Episcopale per la fede l'annuncio e la catechesi in Italia, (d'ora in avanti

3 Discorso del Santo Padre all'apertura dei lavori della 66ma Assemblea Generale della Conferenza Episcopale Italiana (CEI), 19.05.2014, https://press.vatican.va/content/salastampa/it/ bollettino/pubblico/2014/05/19/0360/00804.html (3.04.2015).

4 Cf. $I G, 1-3$.

5 Ufficio Catechistico Nazionale, Il rinnovamento della catechesi. Documento base per la redazione dei catechismi Episcopato Italiano, Roma, 2 febbraio 1970 (d'ora in avanti DB). 
oltre quarant'anni e pubblicato nel $1970^{6}$. Il lavoro di preparazione avvenne con un'ampia consultazione di esperti e incaricati diocesani, e fu approfondito in Convegni catechistici regionali e in ripetute Consulte nazionali dell'Ufficio Catechistico Nazionale ${ }^{7}$.

\section{II senso dei nuovi Orientamenti}

L'obiettivo degli Orientamenti è quello di far risaltare le modalità all'annuncio e i criteri per far accogliere il Vangelo di Gesù. La Chiesa Italiana e i vescovi continuano a ripetere che è necessario annunciarlo con gioia, come avveniva nell'antica Chiesa di Tessalonica ${ }^{8}$. L'impegno dell'annuncio e della catechesi ha sempre caratterizzato il cammino della Chiesa in Italia e per quel che riguarda l'evangelizzazione, Papa Francesco così si esprime: «La Chiesa non evangelizza se non si lascia continuamente evangelizzare. È indispensabile che la Parola di Dio diventi sempre più il cuore di ogni attività ecclesiale. La Parola di Dio ascoltata e celebrata, soprattutto nell'Eucaristia, alimenta e rafforza interiormente i cristiani e li rende capaci di un'autentica testimonianza evangelica nella vita quotidiana. (...). Lo studio della Sacra Scrittura dev'essere una porta aperta a tutti $\mathrm{i}$ credenti. È fondamentale che la Parola rivelata fecondi radicalmente la catechesi e tutti gli sforzi per trasmettere la fede $)^{9}$. Le parole dell'Esortazione di papa Francesco si inseriscono bene nell'attuale contesto socio-culturale in cui la secolarizzazione, il pluralismo culturale, etnico e religioso sono nuove sfide del nostro tempo che non possiamo ignorare. La situazione attuale in Italia sprona i cristiani alla ricerca di Dio, a testimoniare la fede e la ragione della propria fede, e ad annunciare il Vangelo ${ }^{10}$.

6 Cf. B. Forte, Un decalogo per rinnovare la catechesi a 40 anni dal Documento di Base. Conclusioni del Seminario, "Catechesi" 79 (2009/2010) 6, p. 64-65; Ufficio Catechistico Nazionale (d'ora in avanti UCN).

7 Cf. CEDAC, Annuncio e catechesi per la vita cristiana. Lettera alle comunità, ai presbiteri e ai catechisti nel quarantesimo del Documento di base II rinnovamento della catechesi, 4 aprile 2010.

Cf. Questo frammento della lettera ai Tessalonicesi è inserito come il motto introduttivo del documento Incontrare Gesù - 1 Ts 1, 1-5.

9 Francesco, Esortazione apostolica Evangeli gaudium (d'ora in avanti $E G$ ), 24 novembre 2013, nn. 174-175.

${ }^{10}$ Si può aggiungere qui il documento della CEI in cui si propongono gli orientamenti pastorali, sottolineando l'aspetto educativo. Cf. Conferenza Episcopale Italiana, Educare alla vita buona del 
Va accolta anche la sfida delle «culture urbane» in continuo mutamento, amplificato dai mezzi di comunicazione e salvaguardato il rispetto dei vari stili di vita della «cultura rurale» nella quale si sono formate numerose strutture pastorali. Di fronte a questi cambiamenti va rafforzato il rapporto tra conoscenza ed esperienza di fede, ripensando la pastorale e in generale l'azione ecclesiale ${ }^{11}$. Sembra anche importante riferirsi nuovamente al Concilio Vaticano II il quale - già allora - parlò di cambio di mentalità per un nuovo annuncio del vangelo e per la catechesi agli uomini e alle donne. I passaggi indicati nel documento indicano la gradualità come metodo per attuare un processo che aiuti le persone ad approfondire la propria vita cristiana e ciò in linea con quanto indicato nel Rinnovamento della Catechesi ${ }^{12}$, di cui si rivivono oggi gli echi.

Tenendo presenti i cambiamenti socio-culturali e religiosi che interpellano anche la Chiesa, il documento Incontriamo Gesù vuole proporre scelte significative, indicando agli operatori pastorali alcune linee operative per formare alla fede. Gli Orientamenti sollecitano una riflessione sull'annuncio, sul Battesimo e sulla catechesi per ogni periodo della vita, e un invito a ricomporre a livello nazionale le esperienze finora sperimentate nelle diverse diocesi, per mostrare un'unità catechistica nell'azione educativa. In questa linea il documento propone piste su cui riflettere per realizzare progetti innovativi diocesani e parrocchiali relativi all'annuncio e alla catechesi ${ }^{13}$.

\section{Presentazione del testo del documento}

Il documento si apre con la presentazione del Presidente della Conferenza Episcopale Italiana Cardinale Angelo Bagnasco che ne indica le finalità per orientare la pastorale catechistica aiutandola a ridefinire i suoi compiti all'interno dell'azione evangelizzatrice della Chiesa, da intendere come orizzonte e processo.

Vangelo. Orientamenti pastorali dell'Episcopato italiano per il decennio 2010-2020 (d'ora in avanti EVBV), 4 ottobre 2010, nn. 7-11.

11 Cf. $E G$, nn. 25-27.

12 Cf. Insegnamenti di Paolo VI, Vol. IV (1966), Città del Vaticano 1967, p. 304.

13 Cf. Documenti Ufficiali della Santa Sede 1982-1983, Il codice di diritto canonico, Edizioni Dehoniane Bologna, Bologna 1995, cann. 773-780. Il Direttorio Generale per la Catechesi richiede un progetto diocesano di catechesi nei nn. 274-275, mentre al n. 225 indica la programmazione catechistica e la sua armonizzazione con il progetto diocesano tra i compiti espliciti del parroco. 
Segue l'introduzione Con la potenza dello Spirito Santo ${ }^{14}$ che richiama le comunità cristiane ad una «fede operosa», una «carità disinteressata» e una «ferma speranza».

Il corpo del documento si compone di quattro capitoli.

Il primo Abitare con speranza il nostro tempo ${ }^{15}$, cerca di contestualizzare l'azione evangelizzatrice nella «cultura contemporanea come via di missione e non solo come ostacolo» senza tacere tuttavia, le "esigenze di conversione". In primo piano, c'è l'invito specifico ad evangelizzare, partendo dalla vita buona del Vangelo e dall'avventura della fede della Chiesa da realizzare secondo il triplice compito: 1. Annuncio della Parola di Dio (kerygma); 2. Celebrazione dei Sacramenti (liturgia), 3. Servizio della carità (diakonia). Il capitolo termina con un richiamo alla responsabilità dei soggetti dell'annuncio e della catechesi.

Il secondo capitolo, Annunciare il Vangelo di Gesù, affronta in modo specifico lo stile, le caratteristiche e alcune attenzioni metodologiche del primo annuncio; seguono alcune modalità pastorali. Il capitolo si conclude con alcune Proposte pastorali, tra le quali i "Laboratori sull'annuncio".

Nel terzo capitolo: Iniziare, accompagnare e sostenere l'esperienza di fede. Il cammino della Iniziazione cristiana ${ }^{16}$, la centralità è data alla catechesi a servizio dell'iniziazione, uno dei principali impegni delle comunità cristiane. Dopo averla collocata sullo sfondo della «maternità» della Chiesa, si riflette sull'iniziazione cristiana degli adulti e sull'ispirazione catecumenale degli itinerari di fanciulli e ragazzi. Il Capitolo si conclude con alcune proposte pastorali e con l'immagine di Maria, Giuseppe e Gesù al tempio.

Il capitolo quarto, Testimoniare e narrare. Formare servitori del Vangelo ${ }^{17}$ è il capitolo più innovativo riguardante il ministero e la formazione degli evangelizzatori e catechisti. Esso richiede una pluralità di ministeri, di compiti e di figure educative: il vescovo, i presbiteri, i diaconi, il catechista. Un ruolo importante hanno i consacrati e i laici, particolarmente i genitori, i padrini e le madrine. Largo spazio è lasciato alla riflessione sull'identità e sulla vocazione del catechista e del suo radicamento nel compito ricevuto dal vescovo. Della figura del catechista si sottolineano gli ambiti della sua formazione e delle diverse strutture del suo servizio. Il capitolo si conclude con la gioia dello

\footnotetext{
14 Cf. 1 Ts $1,5 \mathrm{a} ; I G 1-7$.

15 Cf. $I G$ 8-31.

16 Cf. $I G 47-62$.

17 IG 63-95.
} 
Spirito Santo ${ }^{18}$, che, grazie alla Parola di Dio chiama, opera e trasforma la Chiesa lungo l'anno liturgico.

In appendice agli Orientamenti è stato aggiunto un glossario con finalità didattiche che ne spiega i concetti utili per chi intenda avviare iniziative di formazione.

\section{II dinamismo dell'evangelizzazione: un cammino comune}

Nella tradizione cristiana si è soliti mettere in luce la missione educatrice della Chiesa, "come madre [che] deve educare alla fede"19. Il processo di maturazione consiste essenzialmente in un'azione generativa, capace di generare e accompagnare i propri figli nella fede ${ }^{20}$. In questo modo la Chiesa adotta la stessa pedagogia usata da Dio nella storia della salvezza con la sapiente pazienza di rispettare le tappe della prefigurazione, della preparazione e dell'istituzione del Suo popolo per far crescere i fedeli. Possiamo considerare questa pedagogia come il sentiero della verità, che orienta progressivamente ad una vita piena. Lo stesso principio educativo deve seguirlo la catechesi che, ponendo sempre Gesù Figlio di Dio al centro, ripercorrere il percorso biblico graduale: dalla rivelazione, alla trascendenza, al mistero.

Il Documento dei Vescovi sottolinea fortemente il ruolo della Chiesa nell'educazione alla fede, promuovendo proposte di annuncio in prospettiva missionaria come necessitano i nostri tempi ${ }^{21}$. Il progetto proposto offre alcune linee orientative sull'evangelizzazione per accompagnare e testimoniare la fede dei credenti e dei catechisti in un cammino comune con tutti i fedeli. A sostegno dell'uomo contemporaneo alla ricerca della verità era desiderabile indicare una continuità tra l'annuncio e le aspirazioni umane. Si potevano approfondire maggiormente - ponendo al centro Cristo crocifisso e risorto - l'annuncio, la catechesi, l'esperienza mistagogica in rapporto con l'esperienza umana' ${ }^{22}$.

181 Ts 1,$6 ; I G$ 96-100.

19 Gal, 4, 26.

20 Cf. Catechismo della Chiesa Cattolica (d'ora in avanti CCC), 169: «Essendo nostra Madre, la Chiesa è anche l'educatrice della fede»».

21 Cf. $I G$ 15-18.

22 Cf. Benedetto XVI, San Paolo l'Apostolo delle genti, Libreria Editrice Vaticana-San Paolo, Città del Vaticano-Cinisello Balsamo 2009, p. 81. 
Cristo, "cuore" della fede e dell'annuncio in ogni tempo, è stato riscoperto nella catechesi del «primo annuncio» e del «secondo annuncio».

Il dinamismo dell'evangelizzazione nel testo che stiamo analizzando non pone l'attenzione solo sulla catechesi ai bambini, ma a quella degli adulti, alla pastorale del battesimo e delle prime fasi della vita. Forse per questo il titolo provvisorio del Documento «documento condiviso» voleva dare spazio a tutte quelle nuove linee di pensiero emerse dalle riflessioni degli ultimi anni, per poterle sperimentare e poi sviluppare nei diversi ambiti della Chiesa.

\section{Educare ed evangelizzare nella prospettiva del futuro}

La Chiesa italiana ha accolto la grande sfida dell'evangelizzazione, asserendo con chiarezza che non si può annunciare Gesù senza incontrarlo di nuovo, senza tornare a Lui per ri-ascoltare il primo annuncio pasquale ${ }^{23}$. Il titolo del documento usa il plurale Incontriamo Gesù, non a caso, ma per far comprendere a tutti che la catechesi dovrà sempre più valorizzare la relazione culturale, soprattutto nell'educazione ed evangelizzazione non solo della singola persona, ma di tutta la Chiesa italiana. Il tipo di annuncio proposto dai Vescovi contiene in sé l'aspirazione alla libertà, alla responsabilità per reagire agli stili di vita indotti dal materialismo, consumismo e relativismo. Per questo sarà necessario trovare nuovi modi, tempi, parole per annunciare il vangelo di Gesù e approfondire la spiritualità cristiana, in armonia con lo stile di papa Francesco e con l'apertura della Chiesa: «La Chiesa a volte si è rinchiusa a trasmettere semplici cose, in semplici precetti, mentre la cosa più importante è il primo annuncio: "Gesù Cristo ti ha salvato!"”.

Da queste parole viene la necessità di educare alla spiritualità; è una sfida per la cultura contemporanea per superare una visione orizzontale della vita che porta l'uomo solo alla sfiducia e alla solitudine, all'isolamento e alla paura. L'educazione alla fede nel Cristo orienta verso l'alto e i desideri della vera gioia di eternità. L'impegno educativo è per questo una sfida antropologica che offre motivazioni e orientamenti per riscoprire il vero senso della fede. 


\section{Abbiate la speranza del nostro tempo ${ }^{24}$}

Dal titolo possiamo intuire l'attenzione che la Chiesa ha verso la realtà in cui abita l'umanità odierna, rilevando non solo le difficoltà da superare ma anche quei segni di speranza presenti in essa: una grande sensibilità per i temi legati alla libertà, alla responsabilità personale ed all'interiorità che non va trascurata per guardare avanti con fiducia. Da questo si nota che tutta la riflessione del documento è rivolta alla speranza e alla nuova evangelizzazione, cioè all'annuncio gioioso del vangelo. Ma dove si fonda questa speranza? Su tre pilastri indicati nel documento "rivelazione", "fede", "Chiesa", i quali includono, a loro volta, la fede nella rivelazione di Dio in Gesù Cristo, trasmessa nella Chiesa come testimonianza.

La fede cristiana ha origine dall'incontro con Gesù Cristo, annunciato nella rivelazione attraverso la storia e l'alleanza tra Dio e gli uomini che si realizza ancor oggi nella Chiesa. La rivelazione ha quindi una dimensione storica personale, gratuita, e la nostra fede, che da essa trae la sua forza, diventa autentica solo quando con convincimento possiamo pronunciare: «Io credo nel Dio di Gesù Cristo». Possiamo affermare che avere fede è innanzi tutto credere nell'uomo e nel Dio di Gesù Cristo che dona lo Spirito per agire, patire, amare e sperare secondo la Sua Parola. Non si può comprendere oggi la Chiesa senza fare riferimento alla prima comunità apostolica e post-pasquale nella quale si "abitava" la rivelazione del mistero di Gesù e si testimoniava la fede cristiana. Non può esserci il vangelo senza la Chiesa, cioè senza la fede ecclesiale.

Riflettiamo ora sul rapporto catechesi-fede-cultura, per approfondire le linee indicate dal documento della Chiesa in Italia. La catechesi è la prima tappa iniziale dell'annuncio della fede e della vita ecclesiale capace di aiutare a fare scelte etiche e morali coerenti. In questo scambio reciproco tra educazione cristiana e cultura umana si esprime e si realizza l'identità profonda del cristiano nell'evento unico indicato da Giovanni: "E il Verbo si fece carne e venne ad abitare in mezzo a noi" 25 . In questo senso la dimensione culturale della catechesi in dialogo con la società di oggi, realizza il rapporto proficuo tra ratio e fides come auspicato da Benedetto XVI.

Tenendo conto di queste premesse possiamo, di seguito mostrare alcune linee educative per una catechesi nella sua dimensione culturale. Sembra importante

\footnotetext{
${ }^{24} I G 8-31$.

25 Gv $1,14$.
} 
innanzitutto, un'apertura gioiosa della fede verso l'uomo e il mondo da realizzarsi attraverso la verità nella carità. Senza la carità, la verità è semplice ideologia, senza la verità, la carità è un sentimento emotivo. In questo senso l'educazione non è solo apprendimento intellettuale ma formazione umana e cristiana. Bisogna allora insegnare il catechismo a partire dalla dimensione umana della carità e desiderare di vivere la vita non staccata dalla realtà. È quindi nella pienezza dell'esperienza umana che il cristianesimo e la catechesi si sviluppano, illuminate dal mistero della fede; in questo scambio reciproco si realizza la missione, suscitando lo stupore di una vita rinnovata da Cristo nella Chiesa.

È quanto invita a fare il documento che stiamo analizzando in riferimento alla parrocchia. Al n. 16 leggiamo: «Esistono ampi spazi anche in questo tempo perché il cristianesimo possa continuare a essere il racconto vissuto di un'esperienza liberante di Dio che ci salva, donandoci - nella gioia dello Spirito - suo figlio Gesù, il Risorto ${ }^{26}$. Uno dei luoghi della trasmissione e dell'educazione alla fede è la parrocchia. «È qui infatti che è possibile rivolgere l'annuncio della buona notizia a tutti, anche ai più deboli, come le persone disabili e le loro famiglie, gli immigrati, i poveri; è qui soprattutto che i cristiani vivono l'anno liturgico, imperniato sulla Domenica, memoria viva della Pasqua $»^{27}$. A questo proposito W. Kasper constata che il movimento ecclesiale del post - concilio tra parrocchia e catechesi ha conosciuto una partenza faticosa, e poi uno sviluppo inedito in continuità con le linee conciliari ${ }^{28}$. Riferendosi alla parrocchia e alla catechesi che passano sulle linee conciliari dobbiamo dire che sono entrambe strumenti basilari per la maturazione comune di tutti i fedeli; i contenuti della catechesi, arricchiti dalla dimensione pedagogica ed ecclesiale, hanno una ricaduta positiva sui credenti in quanto incarnati nella realtà quotidiana. Le comunità parrocchiali devono sentire la necessità della trasmissione della fede per ritrovare il senso profondo della Chiesa e delle persone ad essa affidate e non ridursi ad un'istituzione burocratica "senza vita". Di qui il richiamo dei Vescovi a trasformare le istituzioni ecclesiali per rendere possibile la trasmissione della fede evangelizzando nella cultura.

\footnotetext{
$26 I G 16$.

$27 I G 55$.

28 Cf. W. Kasper, Tornare al primo annuncio. Relazione al Congresso Europeo dei Vescovi e responsabili per la catechesi del CCEE, "Il Regno-Documenti" 54 (2009), p. 336-343.
} 
Procedendo con la riflessione, l'attenzione è posta sui laici e sul loro compito nell'annuncio e nella catechesi attraverso le «alleanze educative $»^{29} \mathrm{e} \mathrm{la}$ capacità di lavorare in rete, per una maggiore efficacia e per rispondere alle sfide in maniera adeguata. Un grande ruolo è riconosciuto alle aggregazioni laicali che si assumono la responsabilità educativa, ciascuna con i propri carismi, con opportuni itinerari che orientano verso una maturazione umana e spirituale. Nel gruppo, la persona trova la facilità di aprirsi, e riesce creare buone relazioni sociali, correndo però il rischio di perdere di vista la dimensione dell'appartenenza a un'associazione o a movimenti. Si deve allora aiutare a ravvivare il sentimento religioso, attraverso momenti di spiritualità per «far emergere la forza educativa della fede verso la pienezza della relazione con Cristo nella comunione ecclesiale» ( $E V B V 53$ ), valorizzando le potenzialità educative. In questo punto nel documento si tratta dei percorsi che si devono integrare nella pastorale ${ }^{30}$.

Il documento non poteva sorvolare sull'importanza della Scrittura, celebrata nella Messa e testimoniata nella carità quando la comunità cristiana si raduna in chiesa per ascoltare la parola di Dio. Ma la Parola proclamata nella liturgia non va accolta solo con la mente ma soprattutto con il cuore, come parola viva che trasforma, perché nella celebrazione eucaristica diventiamo, in Cristo, come dice Paolo, un solo corpo ${ }^{31}$. La liturgia stessa ha poi un potenziale educativo per realizzare un'azione evangelizzatrice "gioiosa" nella quotidianità per far progredire il bene.

Nel documento si sottolinea la centralità di Cristo nella catechesi che deve essere presentata come un'esperienza viva ${ }^{32}$, che prende ispirazione dagli apostoli; l'annuncio della croce gloriosa di Cristo $^{33}$ è per gli operatori pastorali il centro vitale dell'annuncio e come dimensione del servizio. Lo scopo della catechesi è far affiorare il soffio creativo dello Spirito Santo ${ }^{34}$, con l'annuncio della dottrina, con la parola di Dio vissuta e pregata nella liturgia comunitaria e personale. Di tutto deve essere consapevole il catechista svolgendo il compito affidatogli dalla Chiesa e maturando scelte adeguate in campo pedagogico:

\footnotetext{
$29 \quad E V B V 35$.

30 Cf. $E G$ 29; 104.

31 Cf. 1 Cor 10, 16-17.

32 Cf. 2 Cor 2, 2.

33 Cf. Gv 19, 30

${ }^{34}$ Cf. Gv 7, 39.
} 
«Noi infatti non annunciamo noi stessi, ma Cristo Gesù Signore [...] perché "Rifulga la luce dalle tenebre", per far risplendere la conoscenza della gloria di Dio sul volto di Cristo»» ${ }^{35}$.

\section{Annunciare il Vangelo di Gesù}

Uno dei temi più interessanti affrontati dagli Orientamenti per l'annuncio e la catechesi in Italia ${ }^{36}$ riguarda il rinnovamento pastorale da realizzarsi con l'annuncio, elemento qualificante della pastorale contemporanea, e con la catechesi come processo formativo che dura tutta la vita. «Annuncio» e «catechesi» sono due termini interdipendenti fra loro ma con compiti differenti per una finalità comuni «nell'esperienza concreta i momenti distinti dell'azione evangelizzatrice si richiamano costantemente ${ }^{37}$. L'annuncio genera la fede che la catechesi fa crescere e porta a maturazione ${ }^{38}$.

Gli Orientamenti offrono delle riflessioni originali sul rapporto tra annuncio e catechesi. La catechesi è vista come dinamismo relazionale con una connotazione cristocentrica $^{39}$ rivolta alla persona, protagonista libera e responsabile del dialogo ${ }^{40}$. La catechesi come «momento essenziale» del percorso formativo ${ }^{41}$, di cui l'annuncio è «primo» e fondante; ma tutti e due sono fondamentali nel processo di evangelizzazione ecclesiale ${ }^{42}$, inteso come «sorgente, itinerario e traguardo della catechesi e, più ancora, di ogni prassi pastorale» ${ }^{43}$. Il primo annuncio chiama alla conversione attraverso l'incontro con Gesù ${ }^{44}$ a cui «segue la catechesi che fa maturare la conversione iniziale in ordine a una vita cristiana adulta ${ }^{45}$ in un impegno educativo di «accompagnare la maturità della fede» ${ }^{46}$.

\footnotetext{
352 Cor 4, 5-6.

${ }^{36} \mathrm{Cf} . I G 5$.

37 IG 27.

38 Cf. DGC 49; 61.

39 Cf. $I G 27 ; 32$.

40 Cf. $I G 24$.

${ }^{41}$ Cf. $I G 21$.

42 Cf. $I G 19$.

$43 I G 21$.

44 Cf. $I G 20$.

45 Cf. $I G 21$.

${ }^{46}$ Cf. $I G$ 22-27.
} 
II documento orienta alla pratica senza però offrire indicazioni che possano aiutare ad unificare le varie riflessioni ${ }^{47}$.

Sarebbe stato auspicabile prendere in considerazione le varie dimensioni della vita umana per un approfondimento della fede incarnata nella quotidianità. La vita infatti è sorpresa, utopia, disincanto, gratuità, fragilità, gioia e dolore. Proprio in questi spazi emozionali si inscrive il «dibattito» che provoca la domanda su Dio. In tale contesto, la Chiesa oggi è chiamata a ricercare con audacia e creatività nuovi spazi e metodi per rilanciare un nuovo umanesimo, per dare risposte credibili al senso della vita e far comprendere che la storia dell'uomo è abitata da Dio. Una Chiesa in uscita, per usare l'espressione di Papa Francesco verso l'umanità tutta ${ }^{48}$, abbandonando il comodo criterio pastorale del "si è fatto sempre così'. Senza dubbio l'argomento non è nuovo, ma, oggi, è necessario rilanciarlo rimettendo al centro e nella coscienza di molti, anche credenti, il principio dell'«incarnazione». La profonda crisi antropologica, la cultura caratterizzata dall'indifferenza, dal relativismo e dal soggettivismo rendono arduo credere in Dio, fondamentalmente perché si fa fatica a credere nell'uomo. Questa umanità sembra non essere più «capace» di portare in sé il divino. Credere in un Dio incarnato, è possibile solo in una comunità credente e attraverso un processo in grado di valorizzare tutto l'uomo. Per crescere in umanità, Dio deve camminare accanto a noi fianco a fianco ogni giorno e la fede deve essere una risorsa cui attingere per realizzare una autentica integrazione tra fede e vita.

Per riscoprire la centralità di Cristo nella vita è necessario che gli Uffici Catechistici ri-scoprano l'«apostolato biblico» come specifico settore della pastorale diocesana riferita alla formazione di tutti coloro che operano nelle molteplici attività ecclesiali. A questo scopo è auspicabile estendere il Servizio dell'Apostolato Biblico dell'Ufficio Catechetico Nazionale ${ }^{49}$ a specifici settori: 1. Agli animatori biblici con lo scopo di approfondire la fede cristiana alla luce del testo biblico per favorire e sviluppare una spiritualità salda e un discernimento personale e comunitario sulle scelte da compiere di fronte a problemi sempre nuovi; 2. Alla catechesi perché sappia manifestare la sua identità biblica ${ }^{50}$.

47 Cf. A. Fossion, Proposta della fede e primo annuncio, "Catechesi" 78 (2008/2009) 4, p. 30.

48 Cf. $E G 33$; EVBV 13-15.

49 Cf. Ufficio Catechetico Nazionale - Servizio dell'Apostolato Biblico (d'ora in avanti SAB), L'apostolato biblico nelle comunità ecclesiali. Orientamenti operativi, 4-6 febbraio 2005; C. Bissoli (a cura di), L'Animatore Biblico. Identità, competenze, formazione, Elledici, Leumann 2000.

50 Cf. UCN - SAB, Bibbia e catechesi. Come realizzare la formazione biblica alla luce del Progetto Catechistico Italiano, Elledici, Leumann (To) 1999. 
Una valida e sperimentata modalità di annuncio è il laboratorio sostenuto dall' $\mathrm{UCN}^{51}$ inteso come uno stile da assumere per rinnovare gli aspetti dell'azione pastorale non sempre adeguata ai cambiamenti culturali e sociali in atto. La formula laboratoriale intende infatti modificare la prassi, creando un nuovo modello di annuncio capace di intercettare le persone nella vita in un «orizzonte [che] deve essere realmente aperto ${ }^{52}$. Secondo Papa Francesco, i laboratori devono mettere in luce un annuncio di fede a partire dalla realtà socio-religiosa di un territorio. Bisogna determinare i tratti della figura di Gesù e del suo messaggio, particolarmente la capacità di interpretare la vita delle persone, mostrando Gesù come la felicità per ciascuno ${ }^{53}$. Gli Orientamenti invitano ad utilizzare, per raccontare le parole della Scrittura, forme di comunicazione nuove come il web, l'arte, ecc. e, per far questo, come auspica il Papa, bisogna avere il coraggio di trovare nuovi segni, nuovi simboli, un nuovo modo per la trasmissione della Parola: le diverse forme di bellezza che si manifestano in vari ambiti culturali, comprese quelle modalità non convenzionali di bellezza, che possono essere poco significative per gli evangelizzatori, ma che sono diventate particolarmente attraenti per gli altri ${ }^{54}$. È un invito a non aver paura di sperimentare anche percorsi inediti, nella fiducia che lo Spirito non farà mancare il suo supporto e la sua presenza.

\section{Iniziazione cristiana e sfida pastorale}

L'iniziazione cristiana è l'attività che la Chiesa esprime come atto generativo della fede, proprio questa espressione ha motivato i vescovi a dare un ampio spazio negli Orientamenti per l'annuncio e la catechesi in Italia all'iniziazione cristiana $^{55}$, trattata particolarmente nel terzo capitolo. La riflessione prende atto che la Chiesa che non sa più generare alla fede, anche se fa tante altre cose, è una Chiesa sterile e «vecchia», per questo popone la conversione in senso missionario, un cammino di rinnovamento di annuncio e di testimonianza

51 Cf. $I G 46$.

52 Francesco, La mia porta è sempre aperta. Una conversazione con Antonio Spadaro, Rizzoli, Milano 2013, p. 46.

53 Cf. $I G 35$.

54 Cf. Il film Credo. Film d'arte e di nuova evangelizzazione, di M. Camattari, realizzato per l'Anno della Fede con il patrocinio di alcuni uffici nazionali della CEI, Pardes Edizioni.

55 Cf. IG 47-62. 
dell'esperienza cristiana ${ }^{56}$. Tutto ciò non è sufficiente per realizzare un vera conversione, si postula pertanto un processo catecumenale per un «secondo annuncio» necessario per una nuova adesione a Cristo per i genitori, le famiglie e gli operatori pastorali in quanto non sono "tabula rasa" ${ }^{57}$. Una simile situazione riguarda i percorsi di pastorale pre e post-battesimale e per le prime fasi di età. In questo processo ogni comunità cristiana è chiamata a qualificarsi nel progettare e nel vivere un servizio di accoglienza e di accompagnamento nel cammino di fede e di continua conversione di vita dei suoi nuovi membri ${ }^{58}$. Il rinnovamento dell'iniziazione cristiana rende le comunità missionarie, e in particolare le parrocchie, «il luogo in cui tutti i fedeli possono essere convocati per la celebrazione domenicale dell'eucaristia. Nella parrocchia il popolo cristiano si esprime attraverso la vita liturgica durante diverse celebrazioni, che insegnano la dottrina salvifica di Cristo e la pratica della carità del Signore in opere buone e fraterne ${ }^{59}$. L'iniziazione cristiana deve collocare nel suo processo innovativo i vari cammini di catechesi, le celebrazioni sacramentali, le istanze pedagogico-educative, gli elementi rituali, e le tradizioni locali. In questo modo tutta l'attività pastorale sarà spronata a rivedere la sua modalità operativa tenendo conto della cultura, dei processi da attivare, degli strumenti e dei linguaggi con cui comunicare, riti, simboli, ecc. Per attuare tale missione la CEI ritiene fondamentale coinvolgere le famiglie, sottolineando il ruolo educativo dei genitori e dei nonni ${ }^{60}$. Particolare attenzione va riconosciuta al protagonismo dei ragazzi negli itinerari formativi personalizzando l'atto di fede. In questi processi non va mai dimenticata o marginalizzata la situazione di disabilità.

Da questa analisi possiamo dire che gli Orientamenti non sono «rivoluzionari», ma chiedono alle comunità un'azione più chiara nei sacramenti e nella proposta pastorale per i ragazzi (12-14 anni) e per i giovani (15-18 anni). L'iniziazione cristiana deve essere una fida da superare, sempre in modo realistico "senza perdere l'allegria, l'audacia e la dedizione piena di speranza. Non lasciamoci rubare la forza missionaria! ${ }^{61}$.

56 Cf. CEDAC, Annuncio e catechesi per la vita cristiana. Lettera per il quarantesimo del Documento di Base, 4 aprile 2010, p. 10.

57 Cf. Sacra Congregazione per i Sacramenti e il Culto Divino, Rito dell'iniziazione cristiana degli adulti, Edizione Italiana, Roma 1978.

58 Cf. DGC 220.

59 Cf. CCC 2179.

${ }^{60}$ Giovanni Paolo II, Familiaris consortio, 22 novembre 1981, n. 49.

${ }^{61}$ EG 109. 


\section{Formazione dei servitori del Vangelo}

Annunziare la Parola di Dio con gioia significa far emergere la bellezza e la forza del Vangelo, che richiede però un impegno nuovo di fronte ai cambiamenti socioculturali in atto ${ }^{62}$. Nel quarto capitolo degli Orientamenti per l'annuncio e la catechesi in Italia si evidenzia una pluralità di ministeri e servizi per un processo di evangelizzazione ${ }^{63}$ capace di narrare e testimoniare la fede con persone preparate ${ }^{64}$. In primo luogo va riconosciuto il valore dei catechisti, «[...] cresciuti non solo nel numero, ma anche in qualità spirituale, relazionale, pedagogica» ${ }^{65}$; essi diventano «lo spazio di un ampio miglioramento» perché non mancano situazioni di involuzione - catechisti di età avanzata o troppo giovani, situazioni di stagnazione o formazione saltuaria. Questo esige un percorso di maturazione e di crescita della fede dei catechisti, perché siano educatori, accompagnatori e testimoni ${ }^{66}$. Una buona evangelizzazione che porta a $\mathrm{Dio}^{67}$, non può prescindere da un processo formativo umano e motivazionale ${ }^{68}$. La formazione dei catechisti va pensata come un processo personale per una catechesi, che apre «all'altro», verso rapporti umani aperti; una formazione che oltre alla retta dottrina, alla spiritualità e alla fedeltà, deve maturare un'esperienza missionaria ${ }^{69}$. Infatti il fine della formazione del catechista è un processo di crescita personale, e di acquisizione di competenze specifiche religioso-umane ${ }^{70}$ per educare $i$ veri discepoli del Signore e testimoni del suo amore ${ }^{71}$. Il catechista nella sua missione deve tenere presenti alcuni principi guida: «formazione integrata», che sappia distinguere diversi livelli e campi, p.es. far maturare il discepolato delle persone. È ovvio che gli Orientamenti assicurano «la formazione specifica di base a tutti

${ }^{62}$ Cf. $E G 21$.

${ }^{63}$ Cf. $I G 65-66$.

64 Cf. $I G 64$.

65 IG 63 .

${ }^{66}$ Cf. $I G 76$.

${ }^{67}$ Cf. Francesco, Omelia alla Messa per l'incontro dei catechisti in occasione dell 'Anno della Fede, 29 settembre 2013, cit. in $I G 74$.

68 Cf. Ibidem.

69 Cf. $I G 76$.

${ }^{70}$ Cf. DGC 238-245 dedica l'attenzione a tre dimensioni: essere, sapere, saper fare; S. Soreca, La formazione di base dei catechisti, LAS, Roma 2014.

71 Cf. IG 81. 
i catechisti $\rangle^{72}$ e un percorso formativo specifico. Nel documento si parla più volte di «formazione laboratoriale» come impegno per viverlo in collaborazione con gli altri, perché si parla di «formazione collaborativa» per motivare un lavoro comune anche attraverso l'annuncio della Parola ${ }^{73}$. Fare catechesi vuol dire trasmettere una Parola che invita, interroga, crea comunione e salva. Per questo motivo c’è un continuo e abbondante riferimento alla Parola nei vari itinerari del Progetto Catechistico Italiano. È la Parola del Signore che porta alla pienezza della fede, a scoprire il Signore e la propria situazione, ad affidarsi a lui come unico Salvatore. L'annuncio introduce nella storia della salvezza, il cui culmine è la storia di Gesù di Nazareth e attraverso la Parola risuona in modo tale da interpellare ognuno ${ }^{74}$. Per questo il catechista è maestro e testimone dell'ascolto e della lettura personale della Parola, cresce nella conoscenza del Signore e della sua Parola ${ }^{75}$. Il catechista con la conoscenza della Parola, manifesta il Padre, la familiarità del Figlio e il desiderio dello Spirito Santo e fa passare l'amore per il Dio della vita.

\section{Scelta degli operatori pastorali}

Le comunità parrocchiali hanno l'importante compito della scelta degli operatori pastorali. Il primo fra questi è il catechista che deve essere qualificato dal mandato del vescovo. Dalle scelte scaturiscono le responsabilità della formazione e di un'adeguata riflessione nell'ambito teologico. Il sacerdozio battesimale nella vita dell'operatore pastorale fonda il sacerdozio di tutti i credenti, come comunità sacerdotale e profetica. Oltre alla formazione teologica, il catechista deve affinare le sue attitudini spirituali ${ }^{76}$. Esse riguardano la predisposizione a costruire la sua vita e la sua fede personale; esaminare i valori umani e cristiani; condividere la vita della comunità ecclesiale ${ }^{77}$. Il catechista deve orientare la sua vita al servizio facendosi evangelizzatore di coloro che

72 IG 84.

73 Cf. Dei Verbum VI; DCG 45; 112; CT 27; RdC 185-189.

${ }^{74}$ Cf. $I G 3 ; 37$.

75 Cf. Gc 1, 22.

${ }^{76}$ Cf. S. Soreca, La formazione di catechesi per i catechisti. Criteri, competenze e cenni di metodologia, LAS, Roma 2014.

77 Cf. H. Derroitte, Quelques considérations sur la formation des catéchistes. Approches théologique et pédagogique, in H. Derroitte - D. Palmyre (a cura di), Les nouveaux catéchistes. Leur formation, leurs compétences, leur mission, Lumen Vitae, Bruxelles 2008, p. 41-44. 
evangelizza, deve saper mettere in comune la sua fede, essere accogliente. Il catechista propone l'esperienza di Cristo attraverso la condivisione della sua esistenza cristiana, accompagnando la maturazione della fede ${ }^{78}$. Parlando dei catechisti, gli Orientamenti pongono l'accento sul «discernimento in ordine a tale chiamata e al tipo di servizio all'evangelizzazione è pertanto indispensabile: questo compito, ordinariamente, è affidato ai presbiteri, che insieme alla comunità sono chiamati a „riconoscere e promuovere nei fedeli i doni dello Spirito anche in riferimento al servizio della Parola"79. Il servizio fa parte del Progetto comunitario di formazione che favorisce il discernimento e le scelte formative. È uno strumento della comunità per accompagnare il processo di rigenerazione e riaffermazione delle motivazioni fondamentali che aiutano a maturare i carismi educativi e gli itinerari di formazione a un servizio pastorale che genera appartenenza e costruisce comunità ${ }^{80}$.

La scelta degli operatori pastorali coinvolge anche l'UCD con il suo progetto diocesano di catechesi, perché l'organizzazione della pastorale catechistica ha come punto di riferimento il vescovo e la Diocesi, nessuna Chiesa locale può essere priva di un suo Ufficio Catechistico. Tale progetto diocesano di catechesi è guidato dal vescovo che elabora «in modo articolato, coerente e coordinato, i diversi processi catechistici proposti dalla Diocesi $\rangle^{81}$. Questo favorisce l'ascolto e coinvolge le realtà locali. Tutto questo esige un atteggiamento maturo impegnato a conoscere, dialogare e accompagnare nella fede gli uomini di oggi ${ }^{82}$.

\section{Conclusione}

La catechesi e il catechista oggi sono gli argomenti più rilevanti nella Chiesa. Il catechista è un credente che riceve dalla comunità il mandato di proporre a persone credenti e non credenti la fede cristiana, ma anche la coerenza, la sua apertura missionaria, il suo impegno cristiano in famiglia e nel lavoro sono un annuncio silenzioso del vangelo. Nell'annunciare il messaggio cristiano

78 Cf. E. Biemmi, Il secondo annuncio. La grazia di ricominciare, EDB, Bologna 2011, p. 33-41.

79 IG 77.

80 Cf. L. Meddi, «Organizzare la formazione dei catechisti in Italia. Elementi di analisi e prospettive», in Quaderni della segreteria generale della CEI 32 (1998) 2, p. 63.

81 IG 88 .

82 Cf. $I G 12$. 
il catechista terrà sempre presente la finalità educativa della sua azione, che è quella di suscitare stupore, ammirazione, accoglienza, conversione. Egli può accostarsi ai suoi interlocutori con l'esperienza religiosa e la competenza biblica e pedagogica. Annuncia la gioia del vangelo, accompagna le persone dove il Risorto ci precede con la sua iniziativa. Tale prospettiva chiarisce la questione sostenendo che la «capacità evangelizzatrice delle nostre comunità dipende in buona misura dal servizio dei catechisti» ${ }^{83}$, per cui «sarebbe molto difficile per il catechista improvvisare, nella sua azione, uno stile e una sensibilità, ai quali non fosse stato iniziato durante la propria formazione» ${ }^{84}$.

Prendendo il conto di un ruolo importante del catechista e degli operatori pastorali, dei sacerdoti, nel documento si evidenzia una certa omogeneità della situazione culturale, sociale, ecclesiale e pastorale in cui avvengono i processi di formazione ed educazione. Ciò favorisce lo sviluppo dei «bisogni catechistici» ${ }^{85}$ secondo gli aspetti specifici degli orientamenti diocesani e degli orientamenti nazionali; evidenzia la necessità di un confronto al livello interdiocesano, tra formatori e di sostegno reciproco nella verifica dei progetti e delle attività. Per realizzare la formazione dei formatori e dei componenti delle équipe diocesane «con una sapiente opera di coordinamento e patrocinio» ${ }^{86}$ è importante lavorare sulla stessa linea proposta dagli Orientamenti.

\section{Bibliography}

Benedetto XVI, San Paolo l'Apostolo delle genti, Libreria Editrice Vaticana-San Paolo, Città del Vaticano-Cinisello Balsamo 2009.

Biemmi E., Il secondo annuncio. La grazia di ricominciare, EDB, Bologna 2011, p. 33-41.

Bissoli C., (a cura di), L'Animatore Biblico. Identità, competenze, formazione, Elledici, Leumann 2000.

Catechismo della Chiesa Cattolica, 169.

CEDAC, Annuncio e catechesi per la vita cristiana. Lettera alle comunità, ai presbiteri e ai catechisti nel quarantesimo del Documento di base II rinnovamento della catechesi, 4 aprile 2010.
$83 \quad I G 79$.
${ }^{84} I G 86$.
85 DGC 269.
86 IG 90 . 
CEDAC, Annuncio e catechesi per la vita cristiana. Lettera per il quarantesimo del Documento di Base, 4 aprile 2010.

Concilium Oecumenicum Vaticanum II, Costituzione dogmatica sulla divina rivelazione Dei Verbum, (18 novembre 1965), AAS 58 (1966).

Conferenza Episcopale Italiana, Educare alla vita buona del Vangelo. Orientamenti pastorali dell'Episcopato italiano per il decennio 2010-2020, Roma 2010.

Conferenza Episcopale Italiana, Incontriamo Gesù. Orientamenti per l'annuncio e la catechesi in Italia, Edizioni Dehoniane Bologna, Bologna 2014.

Congregazione per il Clero, Direttorio Generale per la Catechesi, LEV, Città del Vaticano 1997.

Derroitte H., Quelques considérations sur la formation des catéchistes. Approches théologique et pédagogique, in: H. Derroitte - D. Palmyre (a cura di), Les nouveaux catéchistes. Leur formation, leurs compétences, leur mission, Lumen Vitae, Bruxelles 2008, p. 41-44.

Discorso del Santo Padre all'apertura dei lavori della 66ma Assemblea Generale della Conferenza Episcopale Italiana (CEI), 19.05.2014, https://press.vatican.va/content/salastampa/it/ bollettino/pubblico/2014/05/19/0360/00804.html (3.04.2015).

Documenti Ufficiali della Santa Sede 1982-1983, Il codice di diritto canonico, Edizioni Dehoniane Bologna, Bologna 1995, cann. 773-780.

Forte B., Un decalogo per rinnovare la catechesi a 40 anni dal Documento di Base. Conclusioni del Seminario, "Catechesi”" 79 (2009/2010) 6, p. 64-65.

Fossion A., Proposta della fede e primo annuncio, "Catechesi” 78 (2008/2009) 4, p. 30.

Francesco, Esortazione apostolica Evangeli gaudium, 24 novembre 2013.

Francesco, La mia porta è sempre aperta. Una conversazione con Antonio Spadaro, Rizzoli, Milano 2013.

Francesco, Omelia alla Messa per l'incontro dei catechisti in occasione dell'Anno della Fede, 29 settembre 2013, cit. in $I G 74$.

Giovanni Paolo II, Esortazione apostolica post-sinodale, Famigliaris Consortio, (22 novembre 1981), AAS 73 (1981), n. 49.

Il film Credo. Film d'arte e di nuova evangelizzazione, di M. Camattari, realizzato per l'Anno della Fede con il patrocinio di alcuni uffici nazionali della CEI, Pardes Edizioni.

Insegnamenti di Paolo VI, Vol. IV (1966), Città del Vaticano 1967, p. 304.

Kasper W., Tornare al primo annuncio. Relazione al Congresso Europeo dei Vescovi e responsabili per la catechesi del CCEE, in "Il Regno-Documenti" 54 (2009), p. 336-343.

Meddi L., «Organizzare la formazione dei catechisti in Italia. Elementi di analisi e prospettive», in Quaderni della segreteria generale della CEI 32 (1998) 2, p. 63.

Sacra Congregazione per i Sacramenti e il Culto Divino, Rito dell'iniziazione cristiana degli adulti, Edizione Italiana, Roma 1978.

Soreca S., La formazione di base dei catechisti, LAS, Roma 2014.

Soreca S., La formazione di catechesi per $i$ catechisti. Criteri, competenze e cenni di metodologia, LAS, Roma 2014.

UCN - SAB, Bibbia e catechesi. Come realizzare la formazione biblica alla luce del Progetto Catechistico Italiano, Elledici, Leumann (To) 1999.

Ufficio Catechetico Nazionale - Servizio dell'Apostolato Biblico, L'apostolato biblico nelle comunità ecclesiali. Orientamenti operativi, 4-6 febbraio 2005. 
The Person and the Challenges

52 Volume 5 (2015) Number 2, p. 33-52

Ufficio Catechistico Nazionale, Il rinnovamento della catechesi Documento base per la redazione dei catechismi Episcopato Italiano, Roma, 2 febbraio 1970. 